\title{
Combined Lifestyle Behaviors and the Incidence of Common Cancer Types in the Norwegian Women and Cancer Study (NOWAC)
}

\author{
Sairah LF Chen $\mathbb{D}^{\prime}$ \\ Tonje Braaten (D) ${ }^{\prime}$ \\ Kristin B Borch (D) \\ Pietro Ferrari $\mathbb{1}^{2}$ \\ Torkjel M Sandanger (D) \\ Therese H Nøst (D) \\ 'Department of Community Medicine, \\ UiT The Arctic University of Norway, \\ Tromsø, Norway; ${ }^{2}$ Nutrition and \\ Metabolism Branch, International Agency \\ for Research on Cancer, World Health \\ Organization, Lyon, France
}

Correspondence: Sairah LF Chen

Department of Community Medicine, UiT The Arctic University of Norway, Hansine Hansens veg 18, Tromsø, 9019, Norway Tel +47 77644786

Email sairah.chen@uit.no
Introduction: Only a small number of studies have examined the impact of combined lifestyle behaviors on cancer incidence, and never in a Norwegian population.

Purpose: To examine linear and nonlinear associations of combined lifestyle factors, assessed through a healthy lifestyle index (HLI), with the incidence of postmenopausal breast, colorectal, lung, postmenopausal endometrial, postmenopausal ovarian, pancreatic, and kidney cancer among women in Norway.

Methods: This prospective study included 96,869 women enrolled in the Norwegian Women and Cancer (NOWAC) cohort. Baseline information on lifestyle factors was collected between 1996 and 2004. The HLI was constructed from five lifestyle factors: physical activity level, body mass index, smoking, alcohol consumption, and diet. Each factor contributed 0 to 4 points to the HLI score, which ranged from 0 to 20 , with higher scores representing a healthier lifestyle. Multiple imputation was used to handle missing data. Cox proportional hazard regression models were used to estimate hazard ratios (HR) and 95\% confidence intervals (CI). Restricted cubic splines were used to examine nonlinearity in the associations.

Results: The HRs for a one-point increment on the HLI score were 0.97 (95\% CI: 0.96$0.98)$ for postmenopausal breast cancer, $0.98(0.96-1.00)$ for colorectal cancer, $0.86(0.84$ $0.87)$ for lung cancer, $0.93(0.91-0.95)$ for postmenopausal endometrial cancer, 0.99 (0.96$1.02)$ for postmenopausal ovarian cancer, $0.92(0.89-0.95)$ for pancreatic cancer, and 0.94 (0.91-0.97) for kidney cancer. Nonlinearity was observed for the inverse associations between HLI score and the incidence of lung cancer and postmenopausal breast cancer.

Conclusion: Based on our results, healthier lifestyle, as assessed by the HLI score, was associated with lower incidence of postmenopausal breast, colorectal, lung, postmenopausal endometrial, pancreatic, and kidney cancer among women, although the magnitude and linearity varied. Adoption of healthier lifestyle behaviors should be a public health priority to reduce the cancer burden among Norwegian women.

Keywords: healthy lifestyle index, cancer prevention, prospective study, composite score

\section{Introduction}

Cancer is the second-leading cause of death worldwide, ${ }^{1}$ with estimated 19.3 million new cancer cases and 10.0 million cancer deaths in $2020 .^{2}$ The latest corresponding numbers in Norway were 34,190 and 11,049 in 2018. ${ }^{3}$ Women accounted for $46 \%$ of the new cases in Norway, where breast cancer remains the most common, followed by colorectal cancer and lung cancer. ${ }^{3}$ According to the latest report from the Cancer Registry of Norway, age-standardized incidence rates 
for most cancers are increasing, as are the number of incident cases. ${ }^{3}$ This trend suggests that the already substantial cancer burden in Norway will continue to grow, placing increasing pressure on the healthcare system in the form of screening and treatment.

It has been estimated by the World Health Organization that $30 \%$ to $50 \%$ of all cancer cases are related to modifiable factors. ${ }^{4}$ Lifestyle factors - namely physical inactivity, overweight and obesity, smoking habits, alcohol consumption, and diet - have been repeatedly identified as cancer risk factors. ${ }^{5,6}$ There is substantial evidence that a large proportion of cancers can be prevented through the adoption of a healthier lifestyle, providing an optimistic avenue for decreasing the future cancer burden. While early diagnosis and treatment for the most common cancers have been improving, they remain a challenge, highlighting the importance of preventive strategies.

Epidemiological studies typically aim to isolate the relationship between single lifestyle factors and cancer risk. While these analyses are critical for identifying novel risk factors and the strength of associations in different populations, ${ }^{7-9}$ they cannot assess the combined impact of several healthy or non-healthy behaviors. An alternate approach is to assess the effects of a combination of lifestyle factors on cancer risk. This concept is increasingly being used to explore the cancerpreventing benefits of an overall healthy lifestyle. Several studies have employed additive exposure scores - either based strictly on recommendations from public health bodies, or based on a combination of recommendations, current scientific knowledge, and sample-specific attributes - referred to as a healthy lifestyle index (HLI). These studies have, for the most part, observed linear risk decreases with increasing increments in their indices. ${ }^{10}$ To our knowledge, nonlinearity in associations between a HLI and cancer incidence has only been explored in one study on lymphoma incidence. ${ }^{11}$

The present study aims to examine the linear and nonlinear associations of combined lifestyle factors, assessed through a score on an a priori-defined HLI, with the incidence of postmenopausal breast, colorectal, lung, postmenopausal endometrial, postmenopausal ovarian, pancreatic, and kidney cancer among women in Norway. To our knowledge, this is the first prospective study to explore associations between a combined measure of lifestyle, including diet, and the incidence of cancer in a Norwegian population.

\section{Materials and Methods}

\section{Study Population and Data Collection}

A detailed description of the Norwegian Women and Cancer Study (NOWAC) has been presented elsewhere. ${ }^{12}$ Briefly, the NOWAC study is a nationwide, prospective cohort study, consisting of approximately 172,000 adult female participants. Women invited to participate in the NOWAC study were randomly sampled from the Norwegian Central Person Register between 1991 and 2007 in multiple sub-cohorts. Those who agreed to participate completed a first self-administered questionnaire. Second questionnaires were sent to all sub-cohorts, except for those enrolled from 2005 to $2007(n=42,671)$, and approximately $70 \%$ of participants responded. All questionnaires collected information on socio-demographic characteristics, reproductive and hormonal factors, selfreported health, physical activity, height, weight, smoking habits, dietary habits, and family history of breast cancer. Questionnaires consisted of either 4 or 8 pages depending on the sub-cohort, with the 8-page questionnaire containing a detailed food frequency questionnaire (FFQ).

The first completed 8-page questionnaire was used as the baseline for the present study. Therefore, sub-cohorts that did not complete an 8-page questionnaire as their first or second questionnaire were excluded $(n=71,210)$, leaving a total of 101,316 women available for this analysis. This number included first questionnaires for sub-cohorts enrolled from 1996 to 1997 (response rate: 57\%) and 2003 to 2004 (response rate: 48\%), and the second questionnaires, administered from 1998 to 1999, for sub-cohorts enrolled from 1991 to 1992. Thus, year at baseline ranged from 1996 to 2004. Women with prevalent cancer, those who died or emigrated before baseline, and those with extreme energy intakes $(<2100$ or $>15,000 \mathrm{KJ} /$ day) were excluded, leaving 96,869 cancer-free participants in the final study sample [see Figure S1 in Supplementary File].

\section{Exposure Assessment and Construction}

To capture overall lifestyle in one measure, relevant lifestyle factors were combined into a HLI, which was a priori based on public health recommendations for cancer prevention from the World Cancer Research Fund/American Institute for Cancer Research (WCRF/AICR) ${ }^{6}$ and current scientific knowledge. Thus, the HLI used for this analysis consisted of five modifiable lifestyle factors - physical activity level, body fatness, smoking status, alcohol consumption, and diet - and each was assigned a score 
ranging from 0 to 4 , with higher scores indicating a healthier lifestyle. Physical activity level was reported by participants on a 10-point scale ranging from not active to very active, where participants were asked to consider the entirety of activity at work, outside work, at home, exercise, and other forms of physical activity. Since this measure could not be categorized by cancer guidelines or other measures of the dose of physical activity, physical activity level was scored by quintile (highest quintile $=4$ through to lowest quintile $=0$ ). Body fatness was assessed by self-reported height (centimeters) and weight (kilograms) to calculated body mass index (BMI, $<23=4,23$ to $<25=3,25$ to $<27=2,27$ to $<30=1, \geq 30=0$ ), smoking status was scored considering intensity and time since cessation (never smoker $=4$, former smoker $>10$ years since cessation $=3$, former smoker $\leq 10$ years since cessation $=2$, smoker $<15$ cigarettes $/$ day $=1$, current smoker $\geq 15$ cigarettes/day $=0$ ), and alcohol consumption was recorded in grams/day (none $=4,>0$ to $<5=3,5$ to $<10$ $=2,10$ to $<20=1,>20=0$ ). To quantify diet, a dietspecific score was generated, which ranged from 0 to 18 , with 18 considered the healthiest diet. Six food groups were included in this score: whole grains, fruit, vegetables, dairy, red meat, and processed meat. Using the Norwegian Weight and Measurement Table, ${ }^{13}$ grams of intake per day were estimated for each food group based on the frequency and portions reported in the FFQ. Each food group was adjusted for energy intake, by dividing grams of intake by daily energy intake, in millijoules (MJ). The energy-adjusted food groups were categorized into quartiles and scored from 0 (lowest quartile) to 3 (highest quartile). Red and processed meat were scored in reverse order. The 18-point diet score was then divided into quintiles for inclusion in the HLI (highest quintile $=4$ through to lowest quintile $=0$ ) $($ Table 1$)$.

The scores for each lifestyle factor were summed to obtain the HLI score, which ranged from 0 to 20 , with a score of 20 being considered the healthiest. Physical activity level, ${ }^{14} \mathrm{BMI},{ }^{15}$ and the $\mathrm{FFQ}^{16,17}$ have been validated in the NOWAC study.

\section{Outcome Assessment}

Women diagnosed with incident cancer after baseline were identified through linkage to the Cancer Registry of Norway, based on codes from the International Classification of Diseases for Oncology, Third Edition (ICD-O-3): ${ }^{18}$ breast cancer [ICD-O-3 code C50], colorectal cancer [C18-20], lung cancer [C34], endometrial cancer [C54], ovarian cancer [C56], pancreatic cancer [C25], and kidney cancer [C64]. Breast, endometrial, and ovarian cancers were considered only among postmenopausal women due to 1) known etiological and risk-factor profile differences between pre- and postmenopausal statuses and 2) inadequate numbers of premenopausal cancer cases. Information on emigration and mortality was obtained through linkage to the Norwegian National Population Register and the national Cause of Death Registry, respectively. The end of the follow-up was 15 December 2018.

\section{Statistical Analysis}

Descriptive statistics were computed using means and standard deviations or medians and interquartile ranges, depending on distribution. Cox proportional hazard regression models were used to estimate hazard ratios (HRs) and 95\% confidence intervals (CIs) for associations between the HLI score and the incidence of postmenopausal breast, colorectal, lung, postmenopausal endometrial, postmenopausal ovarian, pancreatic, and kidney cancer. Age was used as the underlying time-scale, ${ }^{19}$ whereby study entry was defined as age at baseline or age at menopause for postmenopausal breast, endometrial, and ovarian cancer models if the participant was not postmenopausal at baseline. Women were categorized as postmenopausal if they

Table I Healthy Lifestyle Index Scoring System Combining Five Lifestyle Factors Additively

\begin{tabular}{|l|l|l|l|l|l|}
\hline Score & $\begin{array}{l}\text { Physical Activity Level } \\
\text { (10-Point Scale) }\end{array}$ & BMI & Smoking Status & Alcohol Consumption (g/Day) & $\begin{array}{l}\text { Diet } \\
\text { (0-18 Score) }\end{array}$ \\
\hline $\mathbf{0}$ & $1-3$ & $\geq 30$ & Current, $\geq 15$ cig/day & $\geq 20$ & $0-6$ \\
$\mathbf{I}$ & 4 & $27.0-29.9$ & Current, $<15$ cig/day & $10.0-19.9$ & 7 \\
$\mathbf{2}$ & 5 & $25.0-26.9$ & Former, $<10 y r$ since cessation & $5.0-9.9$ & $8-9$ \\
$\mathbf{3}$ & 6 & $23.0-24.9$ & Former, $\geq 10 y r$ since cessation & $>0.0-4.9$ & 10 \\
$\mathbf{4}$ & $7-10$ & $<23$ & Never & 0 & $11-18$ \\
\hline
\end{tabular}

Abbreviations: BMI, body mass index; g/day, grams per day; cig/day, cigarettes smoked per day; yrs, years. 
reported that their menstruation had stopped, reported use of hormone replacement therapy, or if they were $\geq 53$ years of age, to maintain consistency with previous publications from the NOWAC study ${ }^{20,21}$ and the Million Women Study. ${ }^{22}$ Exit time was defined as age at cancer diagnosis, death, emigration, or end of follow-up, whichever occurred first.

The HLI score was first modelled as a continuous variable to estimate HRs corresponding to a one-point increase in the score. Categorical analyses were also carried out by dividing the HLI score into 4 groups (0-5 HLI group, 6-10 HLI group, 11-15 HLI group, and 16-20 HLI group), using the 11-15 HLI group as a reference. The proportional hazards assumption was assessed by Schoenfeld residuals. ${ }^{23}$ The reverse Kaplan-Meier method was used to calculate the median follow-up duration. ${ }^{24}$

The selection of covariates for adjusted models was done on a per-outcome basis, including known risk factors for the exposure, the outcome, or both. ${ }^{25}$ As such, all models were adjusted for education (years) and height (centimeters). Models for postmenopausal breast and postmenopausal endometrial cancer were additionally adjusted for age at menarche (years), use of oral contraceptives (ever, never), parity $(0,1-2,>2)$, breastfeeding (cumulative months $0,<12$ months, $\geq 12$ months), and use of hormone replacement therapy (current, former, never). Models for postmenopausal breast cancer were further adjusted for the history of breast cancer in first-degree relatives (yes, no). The above analyses were conducted on multiple imputed data (described later).

Nonlinear dose-response relationships between the HLI score and the incidence of the included cancer types were modelled with restricted cubic splines. The Akaike information criterion (AIC) was used to evaluate the number of knots and positioning for the best fit. Nonlinearity was assessed through visual inspection of plots and comparison of linear and nonlinear model AIC values.

Several sensitivity analyses were conducted using the HLI score as a continuous variable. To evaluate the driving contributions of each lifestyle factor to the overall observed associations, reduced models were created, each of which excluded one lifestyle factor from the HLI score, thus producing five separate, reduced models for each cancer type. The factor that was excluded from the HLI score was included as a confounder in their respective model, and HRs from these reduced models were compared to those from the full models for each cancer type. This was conducted in both linear and nonlinear models in which the HLI was modelled as a continuous variable. Possible reverse causation was assessed by excluding cancer cases diagnosed within 2 years of baseline. To assess if underweight individuals captured by the healthiest BMI category biased associations for BMI to the null, those with BMI $<18.5$ were excluded. To assess whether the association with colorectal cancer was driven by differing associations of specific sites, associations were also analyzed separately for the incidence of colon and rectal cancer.

\section{Multiple Imputation}

Missing information among covariates was handled using multiple imputation (MI) using chained equations under the assumption that data were missing at random. All covariates required for analysis (lifestyle factors and potential confounders), cancer incidence, and the Nelson Aalen cumulative hazard estimator were included in the MI model. A fully conditional specification was applied, allowing the univariate imputation method and predictors set for each incomplete variable to be specified. ${ }^{26}$ Missing information was replaced with values from $20 \mathrm{MI}$ datasets with five iterations.

MI was performed on physical activity (1-10 scale); weight; height; smoking status; current smoking intensity (number of cigarettes smoked per day on average); time since smoking cessation (year); alcohol consumption (ethanol); daily grams of whole grains, fruit, vegetables, dairy, red meat and processed meat; years of education; and age at menarche (missing $>19 \%$ ). The remaining covariates were complete and therefore only used for prediction purposes. The HLI scores were then generated for each participant in all 20 MI datasets $(96,869$ participants $\times 20$ datasets). HRs and 95\% CIs were estimated by pooling estimates and standard errors from the $20 \mathrm{MI}$ datasets using Rubin's Rule to account for between imputation variance. ${ }^{27}$ All data treatment and statistical analyses were conducted in RStudio Version 1.3.959 with $\mathrm{R}$ Version 4.0.3. ${ }^{28}$

\section{Results}

After a median follow-up of 20.0 years and 15.2 postmenopausal years, there were 4286 postmenopausal breast, 1591 colorectal, 1416 lung, 1043 postmenopausal endometrial, 531 postmenopausal ovarian, 382 pancreatic, and 345 kidney cancer cases diagnosed. The majority (58\%) of participants were in the 11-15 HLI group. The $0-5$ HLI group was the least common $(1 \%)$, while 
the 6-10 (22\%) and 16-20 HLI groups (19\%) were (physical activity level $\geq 6$ ). Mean BMI was 24.7, mean evenly populated. The mean age was 51.6. Overall, alcohol consumption was $1.98 \mathrm{~g} /$ day and the mean diet $49 \%$ of participants were relatively physically active score was 9 (Table 2).

Table 2 Baseline Characteristics of Participants by Healthy Lifestyle Index (HLI) Group, NOWAC Cohort, N = 8I,554, CompleteCase Analyses

\begin{tabular}{|c|c|c|c|c|c|}
\hline & & & HLI & iroup & \\
\hline & All & $0-5$ & $6-10$ & $11-15$ & $16-20$ \\
\hline Number of participants & 81,554 & 878 & 17,847 & 47,435 & 15,394 \\
\hline Number of incident cancer cases & & & & & \\
\hline Postmenopausal breast & 3397 & 39 & 825 & 2014 & 519 \\
\hline Colorectal & 1213 & 18 & 281 & 715 & 199 \\
\hline Lung & 1006 & 33 & 349 & 557 & 67 \\
\hline Postmenopausal endometrial & 807 & 13 & 228 & 444 & 122 \\
\hline Postmenopausal ovarian & 425 & 3 & 101 & 241 & 73 \\
\hline Pancreatic & 284 & 4 & 102 & 136 & 42 \\
\hline Kidney & 268 & 3 & 74 & 156 & 35 \\
\hline Physical activity level $(\%>6)$ & 48.9 & 1.8 & 16.3 & 50.9 & 83.3 \\
\hline BMI, mean (SD) & $24.7(3.9)$ & $30.4(4.2)$ & $27.4(4.5)$ & $24.4(3.4)$ & $22.4(2.2)$ \\
\hline Smoking status, \% & & & & & \\
\hline Never & 36.3 & 0.5 & 13.8 & 35.1 & 68.3 \\
\hline Former & 34.9 & 14.4 & 34.7 & 38.1 & 26.6 \\
\hline Current & 28.8 & 85.1 & 51.5 & 26.8 & 5.0 \\
\hline Alcohol consumption (g/day), median (IQI, IQ3) & $2.0(0.4-5.3)$ & $7.0(2.0-12.2)$ & $3.0(1.0-7.9)$ & $2.0(0.6-5.3)$ & $1.0(0.0-2.9)$ \\
\hline Diet score, median(IQI, IQ3) & $9(7-I I)$ & $6(6-8)$ & $8(6-9)$ & $9(8-10)$ & II (9-12) \\
\hline Age at baseline, mean(SD) & $51.6(6.4)$ & $51.6(5.6)$ & $5 \mathrm{I} .6(6.2)$ & $51.6(6.4)$ & $51.5(6.7)$ \\
\hline Height $(\mathrm{cm})$, mean(SD) & $166.3(5.7)$ & $166.2(5.7)$ & $166.2(5.7)$ & $166.2(5.6)$ & $166.4(5.7)$ \\
\hline Weight $(\mathrm{kg})$, mean(SD) & $68.4(11.5)$ & $84.1(12.6)$ & $75.6(13.1)$ & $67.4(10.2)$ & $61.9(7.1)$ \\
\hline Energy intake (KJ/day), mean(SD) & $7076.9(1900.3)$ & $6602.5(1853.6)$ & $6747.9(1806.3)$ & $7096.3(1866.1)$ & $7425.8(1982.3)$ \\
\hline Education (years), mean(SD) & $12.3(3.4)$ & II.6 (3.I) & $11.9(3.3)$ & $12.3(3.4)$ & $12.9(3.6)$ \\
\hline Age at menarche, mean(SD) & $13.3(1.4)$ & $12.8(1.4)$ & I3.I (I.4) & I3.3 (I.4) & $13.5(1.4)$ \\
\hline Oral contraception use $(\%$ ever) & 54.3 & 59.5 & 58.4 & 54.3 & 48.9 \\
\hline Parity (\%) & & & & & \\
\hline Nulliparous & 8.5 & 11.2 & 8.9 & 8.3 & 8.5 \\
\hline $\mathrm{I}-2$ & 53.4 & 55.6 & 56.8 & 53.5 & 48.9 \\
\hline $3+$ & 38.2 & 33.2 & 34.4 & 38.3 & 42.5 \\
\hline Breastfeeding (\%) & & & & & \\
\hline 0 months & 54.1 & 58.0 & 54.8 & 53.7 & 54.6 \\
\hline $0-12$ months & 24.5 & 29.9 & 27.6 & 24.8 & 19.6 \\
\hline$>12$ months & 21.4 & 12.2 & 17.6 & 21.5 & 25.8 \\
\hline Hormone replacement therapy use (\%) & & & & & \\
\hline Never & 66.7 & 58.1 & 62.5 & 66.5 & 72.3 \\
\hline Former & 11.3 & 17.1 & 12.9 & 11.2 & 9.4 \\
\hline Current & 22.0 & 24.8 & 24.6 & 22.2 & 18.3 \\
\hline
\end{tabular}

Abbreviations: NOWAC, Norwegian women and cancer study; BMI, body mass index; SD, standard deviation; g/day, grams per day; IQ, interquartile; cig/day, cigarettes smoked per day; cm, centimetres; kg, kilograms; KJ, kilojoules. 
Table 3 Linear Associations Between Healthy Lifestyle Index (HLI) Score and Incidence of Common Cancer Types, NOWAC Cohort (1996-2018), $N=96,869$

\begin{tabular}{|c|c|c|c|c|}
\hline & \multicolumn{4}{|c|}{ HLI score } \\
\hline & $0-5$ & $6-10$ & $11-15$ & $16-20$ \\
\hline & Cases $(\mathbf{N})$ & Cases (N) & Cases (N) & Cases (N) \\
\hline & HR (95\% Cl) & HR (95\% CI) & HR (95\% CI) & HR (95\% Cl) \\
\hline Postmenopausal & 41 & 907 & 2162 & 567 \\
\hline Breast $^{a, b, c}$ & I.I3 (0.85-I.50) & $1.12(1.03-1.20)$ & $\mathrm{I} .00$ (ref) & $0.83(0.76-0.91)$ \\
\hline \multirow[t]{2}{*}{ Colorectal $^{a}$} & 19 & 293 & 751 & 218 \\
\hline & $1.46(0.95-2.26)$ & $1.04(0.92-1.19)$ & $\mathrm{I} .00$ (ref) & $0.87(0.75-1.00)$ \\
\hline \multirow[t]{2}{*}{ Lung $^{a}$} & 36 & 376 & 613 & 72 \\
\hline & 3.15 (2.29-4.34) & $1.63(1.45-1.84)$ & $\mathrm{I} .00$ (ref) & $0.39(0.31-0.49)$ \\
\hline Postmenopausal & 13 & 248 & 473 & 128 \\
\hline Endometrial $^{\mathrm{a}, \mathrm{b}}$ & $1.60(0.95-2.69)$ & $1.39(1.20-1.62)$ & $\mathrm{I} .00$ (ref) & $0.78(0.65-0.94)$ \\
\hline Postmenopausal & 3 & 101 & $24 I$ & 73 \\
\hline Ovarian & I.II (0.46-2.67) & $1.06(0.86-1.33)$ & 1.00 (ref) & $0.94(0.73-1.20)$ \\
\hline \multirow[t]{2}{*}{ Pancreatic $^{\mathrm{a}}$} & 4 & 108 & 143 & 45 \\
\hline & $1.49(0.57-3.91)$ & I.87 (I.48-2.37) & 1.00 (ref) & $0.94(0.69-1.28)$ \\
\hline \multirow[t]{2}{*}{ Kidney $^{\mathrm{a}}$} & 3 & 79 & 163 & 37 \\
\hline & $1.26(0.47-3.36)$ & $1.28(0.99-1.67)$ & $\mathrm{I} .00$ (ref) & $0.75(0.53-1.04)$ \\
\hline
\end{tabular}

Notes: ${ }^{a}$ Results from analyses conducted on multiple imputation data, adjusted for education and height. ${ }^{b}$ Additionally adjusted for age at menarche, use of oral contraceptives, parity, breastfeeding, and use of hormone replacement therapy. ${ }^{\mathrm{C}}$ Additionally adjusted for family history of breast cancer in a first-degree relative. Abbreviations: NOWAC, Norwegian women and cancer study; $\mathrm{N}$, number; $\mathrm{HR}$, hazard ratio; $\mathrm{Cl}$, confidence interval.

Results from the MI models (Table 3) showed that the magnitude and direction of the effects were similar to those observed in complete-case analyses [see Table S1 in Supplementary File]. After adjustment for covariates, estimates from the linear analysis using MI data (Figure 1) showed HRs of 0.97 (95\% CI: $0.96-0.98)$ for postmenopausal breast cancer, 0.98 (95\% CI: $0.96-1.00)$ for colorectal cancer, 0.86 (95\% CI: 0.84-0.87) for lung cancer, 0.93 (95\% CI: 0.91-0.95) for postmenopausal endometrial cancer, 0.99 (95\% CI: 0.96-1.02) for postmenopausal ovarian cancer, 0.92 (95\% CI: 0.89-0.95) for pancreatic cancer, and 0.94 (95\% CI: 0.91-0.97) for kidney cancer for every 1-point increase in HLI score. No considerable difference in HRs was observed for colon and rectal cancers (results not shown). When the HLI score was modelled as a categorical variable, HRs for the 16-20 HLI group compared to the 11-15 HLI group were 0.83 (95\% CI: $0.76-0.91)$ for postmenopausal breast cancer, 0.88 (95\% CI: 0.77-1.00) for colorectal cancer, 0.39 (95\% CI:
0.31-0.49) for lung cancer, 0.78 (95\% CI: 0.64-0.94) for postmenopausal endometrial cancer, 0.94 (95\% CI: $0.73-$ 1.20) for postmenopausal ovarian cancer, 0.94 (95\% CI: $0.69-1.28)$ for pancreatic cancer, and 0.72 (95\% CI: 0.52 1.00) for kidney cancer (Table 3).

Analyses of nonlinearity in associations demonstrated that AIC estimates were lowest for all outcomes, except for lung cancer incidence, when three knots at the percentiles were applied, positioned at the defined HLI score boundaries $(1,20)$ and at the HLI score median (13), compared to four- and five-knot models positioned at percentiles or three-, four-, and five-knot models positioned at equal intervals [see Table S2 and Figure S2-8 for plot comparisons in Supplementary File]. The AIC estimate was lowest when five knots positioned at the percentiles were applied to the lung cancer incidence model; however, there were visual indications of overfitting [see Figure S4A in Supplementary File]. Therefore, three knots were applied for all outcomes. The resulting 


\section{Cancer type}

Postmenopausal breast

Colorectal

Lung

Postmenopausal endometrial

Postmenopausal ovarian

Pancreatic

Kidney
$\mathbf{N}$ cases

4286

$0.97(0.96-0.98)$

1281

$0.98(0.96-1.00)$

1097

$0.86(0.84-0.87)$

1043

$0.93(0.91-0.95)$

418

$0.99(0.96-1.02)$

300

$0.92(0.89-0.95)$

282
$0.94(0.91-0.97)$
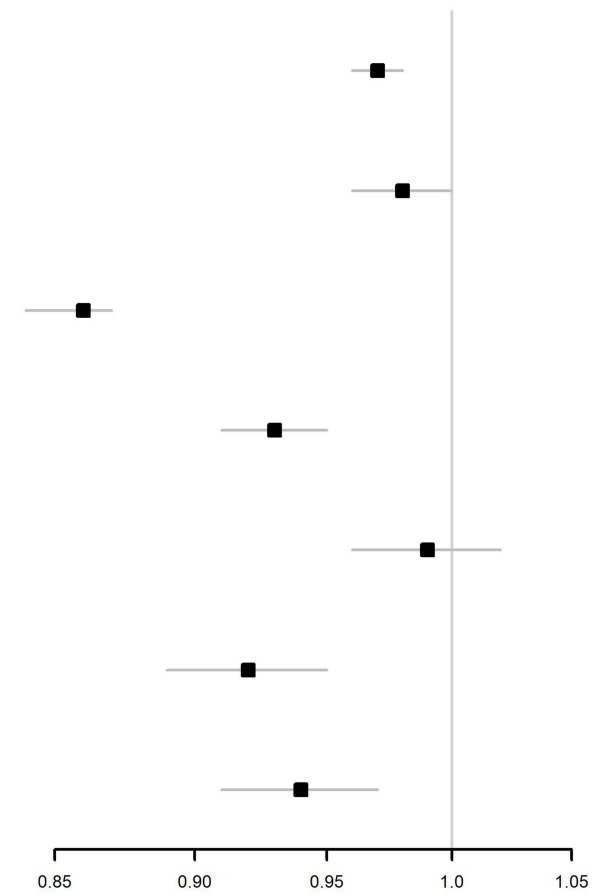

Figure I Forest plot of linear associations between healthy lifestyle index (HLI) score and incidence of postmenopausal breast, colorectal, lung, postmenopausal endometrial, pancreatic and kidney cancers, NOWAC (1996-2018), N =96,869. HRs and 95\% Cls correspond to a I-point increase on the HLI score. Estimates were obtained from multiple imputation data, employed Cox proportional hazard regression, adjusted for education and height.

Abbreviations: NOWAC, Norwegian women and cancer study; HR, hazard ratio; $\mathrm{Cl}$, confidence interval.

plots indicated nonlinearity for lung cancer incidence, with relatively linear decreases in incidence until reaching a plateau at an HLI score of approximately 16 (Figure 2). Nonlinearity was also indicated for postmenopausal breast cancer incidence, with a strengthening of the negative HR gradient at HLI scores above 13 compared to below. The plots for the incidence of colorectal, postmenopausal endometrial, pancreatic, and kidney cancer did not display indications of nonlinearity.

Exclusion of single lifestyle factors from HLI scores did not affect estimates of the incidence of postmenopausal breast, colorectal, and postmenopausal ovarian cancer [see Table S3 in Supplementary File]. For lung cancer incidence, the HR increased to 1.02 (95\% CI: 0.99-1.03) when smoking status was excluded, and the HR decreased to 0.78 (95\% CI: $0.76-0.79)$ when BMI was excluded. For postmenopausal endometrial cancer incidence, the HR decreased to 0.89 (95\% CI: 0.87-0.91) when smoking status was excluded, and the HR increased to 0.97 (95\% CI: 0.95-0.99) when BMI was excluded. For pancreatic cancer incidence, the HR increased to 0.97 (95\% CI: 0.931.01) when smoking status was excluded. For kidney cancer incidence, the HR increased to 0.96 (95\% CI:
0.92-1.00) when BMI was excluded. Among nonlinear models [see Figure S9-15 in Supplementary File], exclusion of BMI and diet resulted in linear associations for postmenopausal breast cancer incidence. Exclusion of smoking status in the nonlinear lung cancer incidence model resulted in no association. Removal of cancers diagnosed within 2 years of baseline and excluding those with BMI $<18.5$ did not alter the results considerably (results not shown).

\section{Discussion}

In this Norwegian national prospective cohort study, we identified inverse associations between our a priori-defined HLI score and the incidence of all included cancer types, except for postmenopausal ovarian cancer. Our examinations indicated that higher HLI scores were associated with lower lung cancer incidence, whereby there were smaller differences in lung cancer incidences among the healthiest participants. There were indications that differences in postmenopausal breast cancer incidence could be greater for women with HLI scores above the median (13). We consider our study population to have a high adherence to 
A Postmenopausal breast cancer

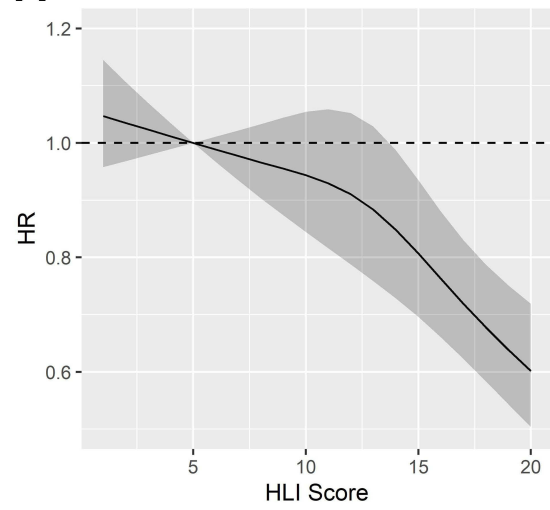

D Postmenopausal endometrial cancer

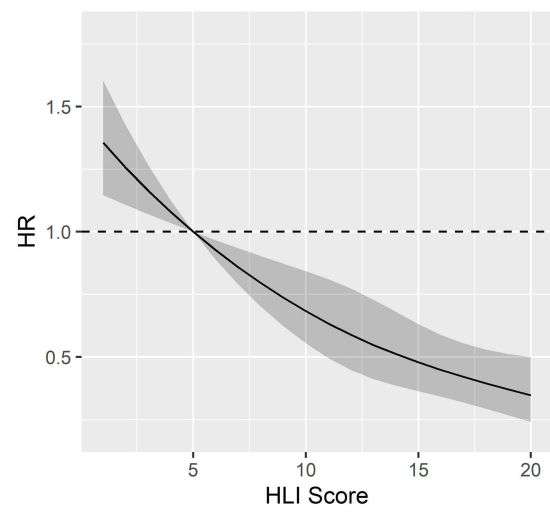

G Kidney cancer

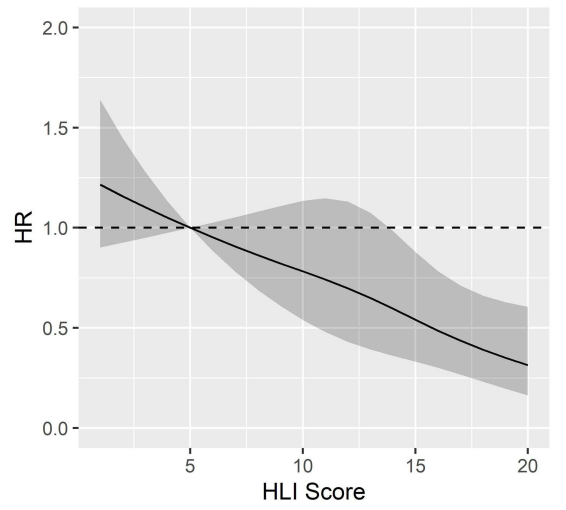

B Colorectal cancer

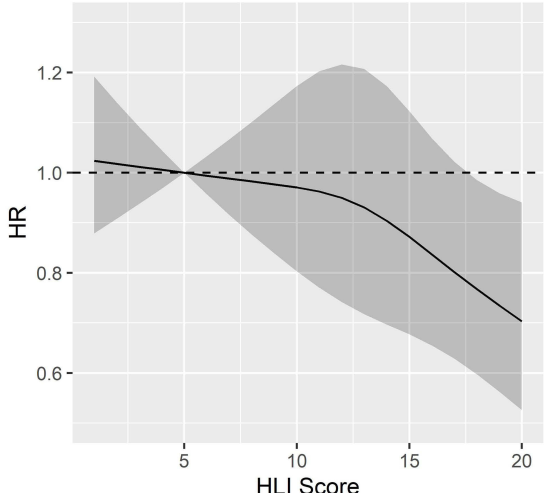

E
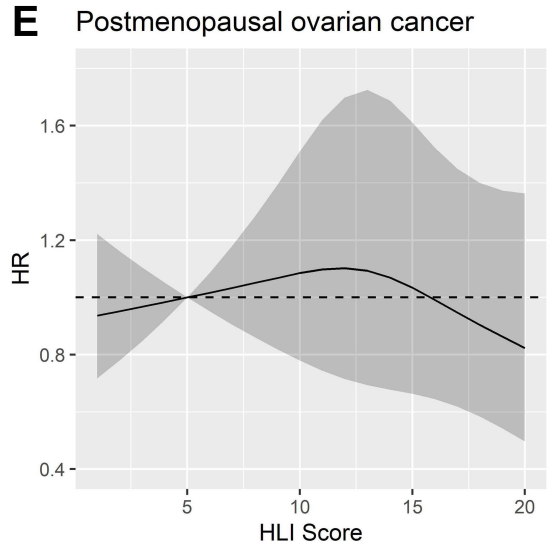

C Lung cancer

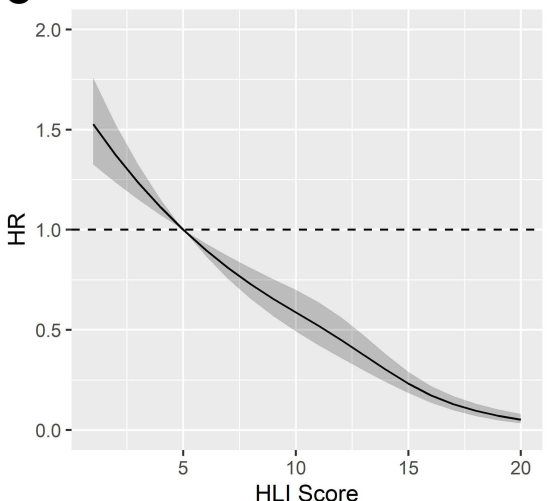

F Pancreatic cancer

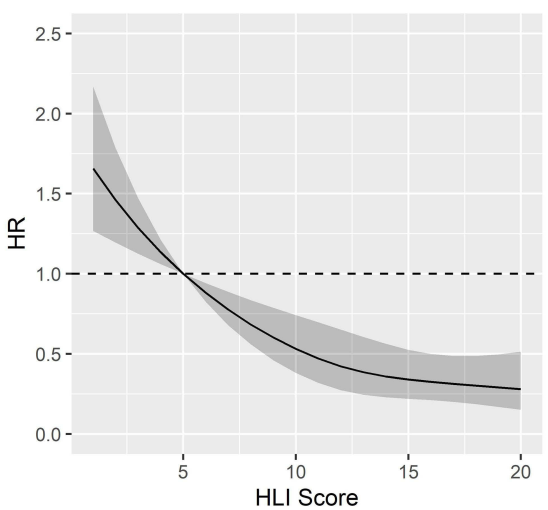

Figure 2 Nonlinear associations between the healthy lifestyle index (HLI) score and incidence of postmenopausal breast, colorectal, lung, postmenopausal endometrial, pancreatic and kidney cancers, NOWAC (1996-20I8), N =96,869. Obtained by applying restricted cubic splines with three knots to the healthy lifestyle index (HLI) score from complete-case analysis data. All models (A-G) were adjusted for education and height. Models (A, D, and E) were additionally adjusted for age at menopause, use of oral contraceptives, parity, breastfeeding, and use of hormone replacement therapy. Model (A) was additionally adjusted for family history of breast cancer in a first-degree relative.

Abbreviations: NOWAC, Norwegian women and cancer study; HLI, healthy lifestyle index; HR, hazard ratio.

healthy lifestyles, with $77 \%$ of participants having HLI scores above 10.

\section{Postmenopausal Breast Cancer}

The incidence of postmenopausal breast cancer decreased by $3 \%$ for every 1 -point increase in HLI score, and our results suggest a nonlinear relationship, based on a comparison of AIC values. Visually, we observed that the inverse association was more pronounced at HLI scores above 13. Our linear estimate is consistent with the results of two other studies, which reported a $3 \%$ and $4 \%$ decrease, respectively, in breast cancer incidence for 
each 1-point increase in HLI score. ${ }^{29,30}$ Other studies on the association between a combined lifestyle measure and breast cancer incidence observed a lower incidence among those with healthier lifestyles. ${ }^{31-36}$ However, these studies are less comparable to ours due to the scoring system employed.

We also conducted multiple sensitivity analyses; each one excluded a different, single lifestyle factor from the HLI score. These analyses resulted in inverse associations that were similar to those observed in the main analyses, suggesting that no specific lifestyle factor drove the observed associations to the HLI score. BMI and diet were suggested as the main contributors to the nonlinear trend in the association, as a linear trend was observed when these factors were excluded. Previous publications from the NOWAC study have observed positive associations between $\mathrm{BMI}^{21}$ and smoking ${ }^{37}$ with cancer incidence; however, there is no evidence from the NOWAC study that physical activity ${ }^{38}$ or food groups and dietary patterns $^{39}$ are associated with postmenopausal breast cancer incidence. Lifestyle factors known to be associated with postmenopausal breast cancer in the NOWAC cohort, namely, BMI and smoking status, did not fully explain our observed association, suggesting that the additive effect of multiple healthy lifestyle factors is important for postmenopausal breast cancer prevention, even though some single lifestyle factors are only weakly associated with cancer incidence.

\section{Colorectal Cancer}

We observed an inverse association between HLI score and colorectal cancer incidence, suggesting a $2 \%$ decrease in incidence for each 1-point increase in HLI score, and a $13 \%$ decrease in incidence when the 16-20 HLI group was compared to the 11-15 HLI group. Allowing for nonlinearity in the HLI indicated that the score was more strongly associated with cancer incidence among women with the highest HLI scores at baseline, similar to our observation for postmenopausal breast cancer risk. However, the large amount of uncertainty in the present study makes it difficult to establish a clear interpretation of this trend, and there are currently no other studies exploring nonlinearity with which we could compare our results. Previous prospective studies that assessed the overall lifestyle with a similar additive exposure score observed comparable effect estimates in linear models, but only with greater precision. ${ }^{40-42}$
Sensitivity analyses indicated that single lifestyle factors did not explain the inverse association we observed. In previous publications of the NOWAC study, clear associations were not observed between single lifestyle factors and colorectal cancer incidence. ${ }^{21,43-45}$ However, our results suggest that healthy lifestyle factors, in sum, are inversely associated, and thus, together, could reduce colorectal cancer incidence.

\section{Lung Cancer}

We observed a strong, inverse association with lung cancer incidence, with a 14\% decrease in incidence for each 1-unit increase in HLI score. This association plateaued at the upper end of HLI scores. To our knowledge, no other study has explored nonlinearity in the relationship between overall lifestyle and lung cancer incidence. Compared to our result, an inverse association of lesser magnitude was observed in the European Prospective Investigation into Cancer and Nutrition (EPIC) cohort, with a $9 \%$ decrease in the incidence of tobacco-related cancers for each 1-unit increase in a similarly constructed 20 -unit index. ${ }^{46}$ This difference in the observed associations could be explained by the fact that several cancer types were pooled in the EPIC analyses. In accordance with this study, a strong inverse association was also observed in a Chinese population; ${ }^{47}$ however, a 5-point HLI and categorical analysis were employed, and thus the strength of associations could not be compared.

Results from sensitivity analyses suggested that smoking status fully explained the observed inverse association. We also observed that smoking status explained the plateau in the nonlinear model. This is consistent with a consensus that smoking is the greatest contributor to lung cancer incidence ${ }^{48,49}$ in the NOWAC cohort and, by design, it is impossible for those with HLI scores above 17 to be current smokers. A study employing American Cancer Society (ACS) recommendations, not including smoking, to construct a combined score also observed a null association with lung cancer incidence. ${ }^{36}$ However, when employing a combined exposure score based on WCRF/AICR recommendations in the EPIC cohort, researchers observed an inverse association between combined lifestyle factors and lung cancer incidence without the influence of smoking. Since dietary factors are weighted heavily in the WCRF/AICR adherence score, carcinogenic effects from a high consumption of preserved and red meat may have been detected. ${ }^{50}$ We observed that a higher BMI was inversely associated with lung cancer 
incidence, which is consistent with the "obesity paradox". 51 While reverse causation due to weight loss associated with the early stages of cancer was ruled out in our study, it is unclear whether the residual confounding effect of smoking cessation could explain the paradoxical observation.

\section{Postmenopausal Endometrial Cancer}

The incidence of postmenopausal endometrial cancer decreased by $7 \%$ for each 1-point increase in HLI score, with no indication of a departure from linearity. Our linear observations are highly consistent with previous studies. A $6 \%$ and $5 \%$ risk decrease per 1-unit increase in HLI, based on 20-unit indices similar to ours, was observed in cohorts from the United States ${ }^{52}$ and Canada. ${ }^{30}$ General adherence to WCRF/AICR and ACS lifestyle recommendations was also inversely associated with endometrial cancer risk in prospective cohort studies. ${ }^{36,53}$

From sensitivity analyses, we observed that the HLI was still inversely associated with postmenopausal endometrial cancer incidence when BMI was excluded from the HLI score, although to a lesser degree than in the main analyses. This suggests that BMI contributed considerably to the association, which is consistent with the known dose-response association between overweight/obesity and cancer risk in the NOWAC study ${ }^{21}$ and with the current consensus that higher BMI is a risk factor for postmenopausal endometrial cancer. ${ }^{54}$ Excluding smoking status from the HLI score strengthened the association, indicating that smoking was protective. While smoking confers risk for most non-communicable diseases, including cancers, cardiovascular disease, and diabetes, its relationship with endometrial cancer risk appears to divert from this pattern. ${ }^{55}$ However, our results suggest that higher physical activity, lower alcohol consumption, healthier diet, and especially lower BMI are, in sum, protective against postmenopausal endometrial cancer incidence. In line with this, higher physical activity levels ${ }^{20}$ and lower $\mathrm{BMI}^{21}$ have been associated with decreased postmenopausal endometrial cancer incidence in the NOWAC cohort. Smoking habits, alcohol consumption, and aspects of diet have not been previously investigated with respect to endometrial cancer in the NOWAC study.

\section{Postmenopausal Ovarian Cancer}

We observed a null association between the HLI score and postmenopausal ovarian cancer incidence. There was no indication of a nonlinear trend in the association. Null associations between comparable HLI scores were also observed in cohorts from Canada, $^{30}$ the United States ${ }^{52}$ and France. ${ }^{34}$ Cohort studies employing scores based on WCRF/AICR and ACS recommendations also observed null associations. $^{36,53}$ As such, there is little evidence in the published literature that overall lifestyle is associated with ovarian cancer incidence, compared to the stronger associations observed for the other cancers we explored.

Sensitivity analyses indicated that no single lifestyle factor suppressed the association. In the NOWAC cohort, ever smoking was not differentially associated with ovarian cancer incidence across histological subtypes and invasiveness, ${ }^{56}$ nor was $\mathrm{BMI}^{21}$ or physical activity ${ }^{57}$ observed to be associated with overall ovarian cancer. This lends further evidence to a minimal or absence of association between lifestyle factors and ovarian cancer incidence in the NOWAC cohort. However, heterogeneity in the etiology of ovarian cancer subtypes may have attenuated the magnitude and reduced the precision of estimates. This should be investigated further in the NOWAC cohort and other populations.

\section{Pancreatic Cancer}

Pancreatic cancer incidence decreased by $8 \%$ for each 1-point increase in HLI score, with no indication of a departure from linearity in the association. To our knowledge, two studies on overall lifestyle and pancreatic cancer have been conducted. Naudin et $\mathrm{al}^{58}$ observed a similar association in the EPIC cohort when employing a 20-unit HLI comparable to ours: risk decreased by $21 \%$ for each 3 -unit increase in the HLI score, corresponding to a decrease of approximately $7 \%$ per 1 -unit increase. In an American cohort, Jiao et $\mathrm{al}^{59}$ also observed an inverse association: compared to the lowest HLI score - 0, the highest score 5 - was associated with a $58 \%$ decrease in pancreatic cancer incidence.

Sensitivity analyses indicated that smoking status fully explained the inverse association we observed between the HLI score and pancreatic cancer incidence. However, Naudin et $\mathrm{al}^{58}$ observed that healthier lifestyles, in addition to smoking habits, were associated with decreased pancreatic cancer incidence in the EPIC cohort. Indeed, obesity and alcohol consumption are also known risk factors for pancreatic cancer, although 
smoking is recognized as the primary modifiable risk factor. ${ }^{60}$ It is possible that we did not observe these associations due to a lack of power or our choice of body fatness measure, since weight gain, not baseline BMI, has been strongly associated with pancreatic cancer risk in the NOWAC cohort. $^{21}$

\section{Kidney Cancer}

Kidney cancer incidence decreased by $6 \%$ for each 1 -point increase in HLI score, with no indication of departure from linearity in the association. To our knowledge, there are no published studies that have examined the association between a similar combined score to ours and kidney cancer incidence to which we can compare our observations. While two other studies based on WCRF and ACS recommendation adherence scores also observed inverse associations, their combined score did not include smoking. ${ }^{36,53}$

Our sensitivity analyses demonstrated that BMI was a strong contributor, in comparison to the other lifestyle factors included the HLI, to the inverse association observed in the main analyses. Indeed, higher BMI has been associated with kidney cancer incidence in the NOWAC study. ${ }^{21}$ Although smoking is a well-established risk factor for kidney cancer, ${ }^{61}$ there was no evidence that it was an especially strong contributor to the association in our study, nor has it been studied as a single risk factor for kidney cancer in the NOWAC study previously. The assessment of other single lifestyle factors in relation to kidney cancer has not been undertaken in the NOWAC study, and these factors, such as physical activity and alcohol consumption, are emerging as important protective or risky behaviors. ${ }^{61}$ Nevertheless, there is evidence from the present study to suggest that the sum of considered lifestyle factors, not just BMI, are important for kidney cancer prevention.

\section{Public Health Implications and Interpreting a Combined Exposure}

The associations between single lifestyle factors and the incidence of different cancer types have been thoroughly examined in previous publications from the NOWAC study. In the present report, we provide an alternate approach for assessing risk, by combining several relevant lifestyle factors into an additive exposure score, as well as exploring linear and nonlinear associations. Given the representativeness of the NOWAC cohort to the
Norwegian population, ${ }^{62}$ we can reasonably suggest that the majority of Norwegian women have high HLI scores, with $77 \%$ of the NOWAC cohort scoring above 10 . Although these women can be considered healthy, our observations suggest that, regardless of baseline HLI score, healthier overall lifestyle, and thus greater adherence to public health recommendations, is protective against postmenopausal breast colorectal, lung, postmenopausal endometrial, pancreatic and kidney cancer. While our findings indicated no added benefit of healthy behaviors, besides smoking reduction for lung and pancreatic cancer, these combined behaviors had a meaningful protective impact on the incidence of postmenopausal breast, postmenopausal endometrial, postmenopausal ovarian, and kidney cancers. Further, limitations in our data may have rendered the remaining associations, without the contribution of smoking, undetectable.

The use of an additive exposure score provides a more holistic assessment of modifiable risk factors compared to single risk factors, as lifestyle behaviors co-exist. We therefore constructed an a priori index based on tangible increments of lifestyle behaviors, as far as our data would allow. With the aim of simplicity, we chose to apply a single, additive index across several cancer types, as this approach offered ease of interpretation, reproducibility across cancer types and populations, ${ }^{63}$ and effective public health messaging. Our results demonstrate that the HLI score is a valuable representation of combined lifestyle factors when evaluating the effects of several modifiable lifestyle factors in a population-based study. For example, the HLI score made it possible to determine whether an overall healthy lifestyle was protective of colorectal cancer, when individual lifestyle factors did not show such associations in the NOWAC cohort.

However, the use of our HLI has its challenges, given the inevitable loss of information that occurs when combining factors into an additive score. We were unable to discern which lifestyle factors specifically contributed to the risk difference at a specific HLI score, nor whether a reduction in one risky behavior could offset the increase in another. ${ }^{7}$ The additive score also assumes linearity in its unit increments, whereas equal distance between units on the HLI may not represent proportional increments of a behavior.

\section{Strengths}

The main strengths of this study are its prospective design, large sample size, long follow-up time, and linkage to 
national registries. An assessment of the external validity of the NOWAC study concluded that the NOWAC cohort is adequately representative of Norwegian women. ${ }^{62}$ Recruitment through random sampling within the Central Population Register of Norway minimized sampling bias. Although education levels in the NOWAC cohort are somewhat higher than the national average, there were no considerable differences in cancer incidence or lifestyle factors compared to national reports. ${ }^{62}$ As such, we can assume that the distribution of participants across HLI scores in the NOWAC study represents the distribution of Norwegian women. Linkage to registries allowed us to be highly confident in the ascertainment of all incident and prevalent cancer cases. Further, MI was used to avoid potential bias created by listwise deletion and to conserve sample size. ${ }^{26}$

\section{Limitations}

This study also has several limitations. Given that lifestyle information is self-reported, it is possible that exposure misclassification introduces bias in our estimates of association. It has been widely acknowledged that research participants tend to underreport their food intake, alcohol consumption, and weight, and overreport variables like height. ${ }^{64}$ Although these tendencies were confirmed in a NOWAC study that compared energy and alcohol consumption in the FFQ to repeated 24-hour dietary recalls, the FFQ still performed well on ranking high and low consumers. ${ }^{17}$ As such, the dietary component of the HLI, which scored participants based on relative intake of food groups, is expected to have achieved an adequate ranking.

Underreporting of weight was also confirmed in a NOWAC validation study, in which the largest tendency to underreport occurred among overweight women, and the largest degree of underreporting occurred among obese women. ${ }^{15}$ Our risk estimates may thus be attenuated due to misclassification of BMI. It is also possible that waist circumference or waist-hip ratio are more accurate indicators of body fatness, and thus metabolic risk, than BMI, ${ }^{65}$ suggesting that stronger associations may have been observed if waist circumference or waist-hip ratio were used. The physical activity report measure was not informed directly by questions pertaining to dose (intensity, frequency, and duration of physical activity). Therefore, differences in physical activity may not have been fully captured, resulting in attenuated estimates. Nevertheless, the physical activity measure demonstrated an adequate Spearman's rank correlation coefficient in the range of $0.36-0.46$ with objective criteria in the validation study and is considered relevant to rank the physical activity levels at a population level. ${ }^{14}$

We did not have information on family history of colorectal, endometrial, pancreatic, and kidney cancers. Due to this and other unmeasured variables, residual confounding may be introduced. Lastly, the models used in this study assumed that lifestyle exposures at baseline were held constant until the participants were censored. Potential changes in lifestyle may be relevant for assessing the relationship between combined lifestyle factors and cancer incidence.

\section{Conclusion}

This is the first prospective study to examine the linear and nonlinear relationship between combined lifestyle factors and the incidence of common cancers in a Norwegian population. Based on our results, healthier lifestyle, as assessed by the HLI score, was associated with lower incidence of postmenopausal breast, colorectal, lung, postmenopausal endometrial, pancreatic, and kidney cancer among women, although the magnitude and linearity varied. The adoption of an overall healthy lifestyle should therefore have a considerable impact on decreasing the cancer burden among women. Limiting smoking is the single most important component of the overall lifestyle for minimizing lung and pancreatic cancer incidence.

\section{Ethics and Consent}

The NOWAC study has been given appropriate approval for collection and handling of questionnaire data by the Regional Committee for Medical and Health Research Ethics (REK) (reference: REK NORD 141/2008) and the Norwegian Data Inspectorate. All participants provided written voluntary and informed consent. The guidelines according to the Declaration of Helsinki were followed.

\section{Acknowledgments}

We would like to acknowledge all the NOWAC participants for their valuable contribution to this study. In addition, thanks are extended to Guri Skeie, Torill E Jensen, and Sabine Naudin for their discussions and input.

Where authors are identified as personnel of the International Agency for Research on Cancer/World Health Organization, the authors alone are responsible for the views expressed in this article, and they do not necessarily represent the decisions, policy or views of the International Agency for Research on Cancer/World Health Organization. 
The publication charges for this article have been funded by a grant from the publication fund of UiT The Arctic University of Norway.

\section{Disclosure}

The authors report no conflicts of interest in this work.

\section{References}

1. GBD. Causes of death collaborators 2017. Global, regional, and national age-sex-specific mortality for 282 causes of death in 195 countries and territories, 1980-2017: a systematic analysis for the Global Burden of Disease Study 2017. Lancet. 2018;392 (10159):1736-1788.

2. Sung H, Ferlay J, Siegel RL, et al. Global cancer statistics 2020: GLOBOCAN estimates of incidence and mortality worldwide for 36 cancers in 185 countries. CA Cancer J Clin. 2021;71(3):209-249.

3. Cancer Registry of Norway. Cancer in Norway 2018: Cancer Incidence, Mortality, Survival and Prevalence in Norway. Oslo: Cancer Registry of Norway; 2018.

4. World Health Organization (WHO). Cancer. Available from: https:// www.who.int/news-room/fact-sheets/detail/cancer. Accessed October 9, 2020.

5. Weiderpass E. Lifestyle and Cancer Risk. J Prev Med Public Health. 2010;43(6):459-471. doi:10.3961/jpmph.2010.43.6.459

6. World Cancer Research Fund/American Institute for Cancer Research (WCRF/AICR). Diet, Nutrition, Physical Activity and Cancer: A Global Perspective: WCRF/AICR; 2018.

7. Friedenreich $\mathrm{CM}$, McTiernan A. Combining variables for cancer risk estimation: is the sum better than the parts? Cancer Prevent Res. 2018;11(6):313-316. doi:10.1158/1940-6207.CAPR-18-0102

8. Pronk NP, Anderson LH, Crain AL, et al. Meeting recommendations for multiple healthy lifestyle factors. Prevalence, clustering, and predictors among adolescent, adult, and senior health plan members. Am J Prev Med. 2004;27(2 Suppl):25-33. doi:10.1016/j. amepre.2004.04.022

9. Anand P, Kunnumakkara AB, Sundaram C, et al. Cancer is a preventable disease that requires major lifestyle changes. Pharm Res. 2008;25(9):2097-2116. doi:10.1007/s11095-008-9661-9

10. Zhang Y-B, Pan X-F, Chen J, et al. Combined lifestyle factors, incident cancer, and cancer mortality: a systematic review and meta-analysis of prospective cohort studies. Br J Cancer. 2020;122 (7):1085-1093. doi:10.1038/s41416-020-0741-x

11. Naudin S, Solans Margalef M, Saberi Hosnijeh F, et al. Healthy lifestyle and the risk of lymphoma in the European prospective investigation into cancer and nutrition study. Int $j$ Cancer. 2020;147 (6):1649-1656. doi:10.1002/ijc.32977

12. Lund E, Dumeaux V, Braaten T, et al. Cohort Profile: the Norwegian Women and Cancer Study-NOWAC-Kvinner og kreft. Int J Epidemiol. 2007;37(1):36-41. doi:10.1093/ije/dym137

13. Landsforeningen for kosthold og helse. Mål Og Vekt for Matvarer (Norwegian Weight and Measurement Table). Oslo; 1995.

14. Borch KB, Ekelund U, Brage S, Lund E. Criterion validity of a 10-category scale for ranking physical activity in Norwegian women. Int J Behav Nutr Phys Act. 2012;9:2. doi:10.1186/1479-5868-9-2

15. Skeie G, Mode N, Henningsen M, Borch KB. Validity of self-reported body mass index among middle-aged participants in the Norwegian Women and Cancer study. Clin Epidemiol. 2015;7:313-323. doi:10.2147/CLEP.S83839

16. Parr CL, Veierod MB, Laake P, Lund E, Hjartaker A. Test-retest reproducibility of a food frequency questionnaire (FFQ) and estimated effects on disease risk in the Norwegian Women and Cancer Study (NOWAC). Nutr J. 2006;5:4. doi:10.1186/1475-2891-5-4
17. Hjartåker A, Andersen LF, Lund E. Comparison of diet measures from a food-frequency questionnaire with measures from repeated 24-hour dietary recalls. The Norwegian Women and Cancer Study. Public Health Nutr. 2007;10(10):1094-1103. doi:10.1017/S1368980007702872

18. World Health Organization (WHO). International Classification of Diseases for Oncology: Third ed. Geneva: Switzerland; 2013.

19. Korn EL, Graubard BI, Midthune D. Time-to-event analysis of longitudinal follow-up of a survey: choice of the time-scale. Am J Epidemiol. 1997;145(1):72-80. doi:10.1093/oxfordjournals.aje.a009034

20. Borch KB, Weiderpass E, Braaten T, Jareid M, Gavrilyuk OA, Licaj I. Physical activity and risk of endometrial cancer in the Norwegian Women and Cancer (NOWAC) study. Int $j$ Cancer. 2017;140(8):1809-1818. doi:10.1002/ijc.30610

21. da Silva M, Weiderpass E, Licaj I, Lissner L, Rylander C. Excess body weight, weight gain and obesity-related cancer risk in women in Norway: the Norwegian Women and Cancer study. $\mathrm{Br} J$ Cancer. 2018;119(5):646-656. doi:10.1038/s41416-018-0240-5

22. Beral V, Banks E, Reeves G, Bull D. Breast cancer and hormone-replacement therapy in the Million Women Study. Lancet. 2003;362(9382):419-427. doi:10.1016/S0140-6736(03)14596-5

23. Schoenfeld D. Partial residuals for the proportional hazards regression model. Biometrika. 1982;69(1):239-241. doi:10.1093/biomet/69.1.239

24. Schemper M, Smith TL. A note on quantifying follow-up in studies of failure time. Control Clin Trials. 1996;17(4):343-346. doi:10.1016/0197-2456(96)00075-X

25. VanderWeele TJ. Principles of confounder selection. Eur J Epidemiol. 2019;34(3):211-219. doi:10.1007/s10654-019-00494-6

26. van Buuren S. Flexible Imputation of Missing Data. 2nd ed. Boca Raton, Florida: Taylor \& Francis Group; 2018.

27. Rubin DB. Multiple Imputation for Nonresponse in Surveys. Hoboken: WILEY; 2009.

28. $\mathrm{R}$ Foundation for Statistical Computing. $R$ : A Language and Environment for Statistical Computing [Computer Program]. Vienna, Austria: R Foundation for Statistical Computing; 2020.

29. Arthur R, Wassertheil-Smoller S, Manson JE, et al. The combined association of modifiable risk factors with breast cancer risk in the women's health initiative. Cancer Prevent Res. 2018;11(6):317-326. doi:10.1158/1940-6207.CAPR-17-0347

30. Arthur R, Kirsh VA, Kreiger N, Rohan T. A healthy lifestyle index and its association with risk of breast, endometrial, and ovarian cancer among Canadian women. Cancer Causes Control. 2018;29 (6):485-493. doi:10.1007/s10552-018-1032-1

31. McKenzie F, Ferrari P, Freisling H, et al. Healthy lifestyle and risk of breast cancer among postmenopausal women in the European prospective investigation into cancer and nutrition cohort study. Int $j$ Cancer. 2015;136(11):2640-2648. doi:10.1002/ijc.29315

32. McKenzie F, Ellison-Loschmann L, Jeffreys M, Firestone R, Pearce N, Romieu I. Healthy lifestyle and risk of breast cancer for indigenous and non-indigenous women in New Zealand: a case control study. BMC Cancer. 2014;14:12. doi:10.1186/1471-2407-14-12

33. Sánchez-Zamorano LM, Flores-Luna L, Angeles-Llerenas A, et al. Healthy lifestyle on the risk of breast cancer. Cancer Epidemiol Biomarkers Prevent. 2011;20(5):912-922. doi:10.1158/1055-9965.EPI10-1036

34. Dartois L, Fagherazzi G, Boutron-Ruault MC, Mesrine S, ClavelChapelon F. Association between five lifestyle habits and cancer risk: results from the E3N cohort. Cancer Prevent Res. 2014;7 (5):516-525. doi:10.1158/1940-6207.CAPR-13-0325

35. Romaguera D, Gracia-Lavedan E, Molinuevo A, et al. Adherence to nutrition-based cancer prevention guidelines and breast, prostate and colorectal cancer risk in the MCC-Spain case-control study. Int $j$ Cancer. 2017;141(1):83-93. doi:10.1002/ijc.30722

36. Kabat GC, Matthews CE, Kamensky V, Hollenbeck AR, Rohan TE. Adherence to cancer prevention guidelines and cancer incidence, cancer mortality, and total mortality: a prospective cohort study. $\mathrm{Am}$ J Clin Nutr. 2015;101(3):558-569. doi:10.3945/ajen.114.094854 
37. Gram IT, Little MA, Lund E, Braaten T. The fraction of breast cancer attributable to smoking: the Norwegian women and cancer study 1991-2012. Br J Cancer. 2016;115(5):616-623. doi:10.1038/ bjc. 2016.154

38. Borch KB, Lund E, Braaten T, Weiderpass E. Physical activity and the risk of postmenopausal breast cancer - the Norwegian Women and Cancer Study. J Negat Results Biomed. 2014;13:3. doi:10.1186/ 1477-5751-13-3

39. Engeset D, Dyachenko A, Ciampi A, Lund E. Dietary patterns and risk of cancer of various sites in the Norwegian European Prospective Investigation into Cancer and Nutrition cohort: the Norwegian Women and Cancer study. Eur j Cancer Prevent. 2009;18(1):69-75. doi:10.1097/CEJ.0b013e328305a091

40. Aleksandrova K, Pischon T, Jenab M, et al. Combined impact of healthy lifestyle factors on colorectal cancer: a large European cohort study. BMC Med. 2014;12:168. doi:10.1186/s12916-014-0168-4

41. Kirkegaard H, Johnsen NF, Christensen J, Frederiksen K, Overvad K, Tjønneland A. Association of adherence to lifestyle recommendations and risk of colorectal cancer: a prospective Danish cohort study. BMJ. 2010;341:c5504. doi:10.1136/bmj.c5504

42. Wang K, Ma W, Wu K, et al. Healthy lifestyle, endoscopic screening, and colorectal cancer incidence and mortality in the United States: a nationwide cohort study. PLoS Med. 2021;18(2):e1003522. doi:10.1371/journal.pmed.1003522

43. Gram IT, Braaten T, Lund E, Le Marchand L, Weiderpass E. Cigarette smoking and risk of colorectal cancer among Norwegian women. Cancer Causes Control. 2009;20(6):895-903. doi:10.1007/ s10552-009-9327-x

44. Oyeyemi SO, Braaten T, Botteri E, Berstad P, Borch KB. Exploring geographical differences in the incidence of colorectal cancer in the Norwegian Women and Cancer Study: a population-based prospective study. Clin Epidemiol. 2019;11:669-682. doi:10.2147/CLEP.S207413

45. Oyeyemi SO, Braaten T, Licaj I, Lund E, Benjaminsen borch K. Physical activity patterns and the risk of colorectal cancer in the Norwegian women and cancer study: a population-based prospective study. BMC Cancer. 2018;18(1):1216. doi:10.1186/s12885-018-5092-0

46. McKenzie F, Biessy C, Ferrari P, et al. Healthy lifestyle and risk of cancer in the European prospective investigation into cancer and nutrition cohort study. Medicine. 2016;95(16):e2850. doi:10.1097/ MD.0000000000002850

47. He Y, Bai Y, Wei S, et al. Healthy lifestyle and cancer risk among Chinese population in the Dongfeng-Tongji cohort. Ann Med. 2020;52(7):393-402. doi:10.1080/07853890.2020.1798017

48. Hansen MS, Licaj I, Braaten T, Lund E, Gram IT. The fraction of lung cancer attributable to smoking in the Norwegian women and cancer (NOWAC) Study. Br J Cancer. 2020;2:548.

49. Malhotra J, Malvezzi M, Negri E, La Vecchia C, Boffetta P. Risk factors for lung cancer worldwide. Eur Respir J. 2016;48(3):889. doi:10.1183/13993003.00359-2016

50. Xue XJ, Gao Q, Qiao JH, Zhang J, Xu CP, Liu J. Red and processed meat consumption and the risk of lung cancer: a dose-response meta-analysis of 33 published studies. Int J Clin Exp Med. 2014;7 (6):1542-1553.
51. Mavridis K, Michaelidou K. The obesity paradox in lung cancer: is there a missing biological link? J Thorac Dis. 2019;11(Suppl 3): S363-s366. doi:10.21037/jtd.2018.12.69

52. Arthur R, Wassertheil-Smoller S, Rohan TE, et al. Associations of a healthy lifestyle index with the risks of endometrial and ovarian cancer among women in the Women's Health Initiative Study. Am J Epidemiol. 2019;188(2):261-273. doi:10.1093/aje/kwy249

53. Romaguera D, Vergnaud AC, Peeters PH, et al. Is concordance with World cancer research fund/American Institute for cancer research guidelines for cancer prevention related to subsequent risk of cancer? Results from the EPIC study. Am J Clin Nutr. 2012;96(1):150-163. doi:10.3945/ajen.111.031674

54. Reeves GK, Pirie K, Beral V, Green J, Spencer E, Bull D. Cancer incidence and mortality in relation to body mass index in the Million Women Study: cohort study. BMJ. 2007;335(7630):1134. doi:10.1136/bmj.39367.495995.AE

55. Zhou B, Yang L, Sun Q, et al. Cigarette smoking and the risk of endometrial cancer: a meta-analysis. Am J Med. 2008;121(6):501508.e503. doi:10.1016/j.amjmed.2008.01.044

56. Licaj I, Jacobsen BK, Selmer RM. Smoking and risk of ovarian cancer by histological subtypes: an analysis among $300 \quad 000$ Norwegian women. $\mathrm{Br} \quad J$ Cancer. 2017;116(2):270-276. doi: $10.1038 /$ bjc. 2016.418

57. Weiderpass E, Margolis KL, Sandin S, et al. Prospective study of physical activity in different periods of life and the risk of ovarian cancer. Int $j$ Cancer. 2006;118(12):3153-3160. doi:10.1002/ijc.21800

58. Naudin S, Viallon V, Hashim D, et al. Healthy lifestyle and the risk of pancreatic cancer in the EPIC study. Eur $J$ Epidemiol. 2020;35 (10):975-986. doi:10.1007/s10654-019-00559-6

59. Jiao L, Mitrou PN, Reedy J, et al. A combined healthy lifestyle score and risk of pancreatic cancer in a large cohort study. Arch Intern Med. 2009;169(8):764-770. doi:10.1001/archinternmed.2009.46

60. Lowenfels AB, Maisonneuve P. Epidemiology and risk factors for pancreatic cancer. Best Pract Res Clin Gastroenterol. 2006;20 (2):197-209. doi:10.1016/j.bpg.2005.10.001

61. Chow W-H, Dong LM, Devesa SS. Epidemiology and risk factors for kidney cancer. Nat Rev Urol. 2010;7(5):245-257. doi:10.1038/ nrurol.2010.46

62. Lund E, Kumle M, Braaten T, et al. External validity in a populationbased national prospective study-the Norwegian Women and Cancer Study (NOWAC). Cancer Causes Control. 2003;14(10):1001-1008. doi:10.1023/B:CACO.0000007982.18311.2e

63. Burggraf C, Teuber R, Brosig S, Meier T. Review of a priori dietary quality indices in relation to their construction criteria. Nutr Rev. 2018;76(10):747-764. doi:10.1093/nutrit/nuy027

64. Willett W. Nutritional Epidemiology. 3rd ed. Oxford: Oxford University Press; 2013.

65. Aleksandrova K, Nimptsch K, Pischon T. Influence of obesity and related metabolic alterations on colorectal cancer risk. Curr Nutr Rep. 2013;2(1):1-9. doi:10.1007/s13668-012-0036-9
Clinical Epidemiology

\section{Publish your work in this journal}

Clinical Epidemiology is an international, peer-reviewed, open access, online journal focusing on disease and drug epidemiology, identification of risk factors and screening procedures to develop optimal preventative initiatives and programs. Specific topics include: diagnosis, prognosis, treatment, screening, prevention, risk factor modification,

Submit your manuscript here: https://www.dovepress.com/clinical-epidemiology-journal systematic reviews, risk \& safety of medical interventions, epidemiology \& biostatistical methods, and evaluation of guidelines, translational medicine, health policies \& economic evaluations. The manuscript management system is completely online and includes a very quick and fair peer-review system, which is all easy to use. 